\title{
An Inverse Scattering Problem for Dirac Equations with Time-Dependent Electromagnetic Potentials
}

\author{
Dedicated to the memory of Professor Nobuhisa Iwasaki
}

By

\author{
Hiroshi T. ITO*
}

\begin{abstract}
We consider an inverse scattering problem, by using a time-dependent method, for the Dirac equation with a time-dependent electromagnetic field. The Fourier transform of the field is reconstructed from the scattering operator on a Lorents invariant set

$$
D:=\left\{(\tau, \xi) \in \mathbb{R} \times \mathbb{R}^{3} ;|\tau|<c|\xi|\right\}
$$

in the dual space of the space-time. As corollaries of this result, we can reconstruct the electromagnetic field completely if it is a finite sum of fields each of which is a time-independent one by a suitable Lorentz transform, and we can also determine the field uniquely if the ficlds satisfics some exponential decay condition. Our assumptions and results are independent of a choice of inertial frames.
\end{abstract}

\section{$\S 1$. Introduction}

In this paper we consider an inverse scattering problem for the Dirac equation with a time-dependent electromagnetic field.

If the field is time-independent and short range, then it can be completely reconstructed from the scattering operator (see [Is], [It], [J]). This means that the field can be determined by scattering experiments in the inertial frame in which the field is time-independent. Because of the relativistic invariance of the Dirac equation, it follows that the field can be also determined by scattering experiments in any inertial frame, the field is in general time-dependent. Thus, it is important to study the Dirac equation with time-dependent fields for the

Communicated by Y. Takahashi, December 26, 1997.

1991 Mathematics Subject Classification(s): 81U40, 81Uxx, 83A05, 35R30

* Department of Computer Science, Ehime University, Matsuyama 790-8577, Japan

e-mail: ito@cs.ehime-u.ac.jp 
inverse scattering problem.

We proceed our argument by fixing an inertial frame; however, our assumptions and results are stated in a way independent of a choice of inertial frames (see Section 5 ). The variable $t$ denotes the time and $x$ is the spacevariable.

We begin with some explanation for our notations. We denote by $\langle a, b\rangle_{R^{a}}$ the usual inner product of $a$ and $b$ in $\mathbb{R}^{d}$ and may write $a \cdot b$ or $\langle a, b\rangle$ for simplicity. Moreover, the usual norm of $\mathbb{R}^{d}$ is denoted by the same symbol $|\cdot|$ for any $d$ if no confusion occures. We also use the symbol $\langle T, u\rangle$ in place of $T(u)$ for a distribution $T$ and a test function $u$.

The Dirac equation with an electromagnetic potential

$$
A=\left(A^{0}, A^{1}, A^{2}, A^{3}\right): \mathbb{R}_{t} \times \mathbb{R}_{x}^{3} \longrightarrow \mathbb{R}^{4}
$$

is given by

$$
\begin{gathered}
i \frac{d}{d t} \Psi(t)=H_{A}(t) \Psi(t), \quad \Psi(t) \in \mathscr{H}:=\mathbb{L}^{2}\left(\mathbb{R}_{x}^{3} ; \mathbb{C}^{4}\right), \\
H_{A}(t)=c \sum_{j=1}^{3} \alpha_{j}\left(D_{j}-A^{j}(t, x)\right)+\alpha_{4} m c^{2}-A^{0}(t, x) I_{4},
\end{gathered}
$$

where $c>0$ is the velocity of light, $m \geq 0$ the rest mass of the particle, $D_{0}=$ $-i \partial / \partial x_{j}$, and $\alpha_{j}$ s are $4 \times 4$ Hermitian matrices with the following properties:

$$
\alpha_{j} \alpha_{k}+\alpha_{k} \alpha_{j}=2 \delta_{j k} I_{4}, \quad 1 \leq j, k \leq 4,
$$

where $\delta_{\jmath k}$ is the Kronecker symbol and $\mathbb{I}_{n}$ is the $n \times n$ identity matrix.

Let $\mathbb{L} \neq\{0\}$ be a subspace of $\mathbb{R}^{4}$. Then we denote by $X_{L}$ the orthogonal projection of $X=(t, x) \in \mathbb{R}^{4}$ onto $\mathbb{L}$ and define a class of potentials:

$$
S(L):=\left\{A \in \mathscr{B}^{1}\left(\mathbb{R}^{4}, \mathbb{R}^{4}\right) ; \int_{0}^{\infty} g_{A}^{L}(r) d r<\infty\right\},
$$

where $g_{A}^{L}(r):=\sup _{\left|X_{L}\right| \geq r}|A(X)|$ and $\mathscr{B}^{1}\left(\mathbb{R}^{4}, \mathbb{R}^{4}\right)$ is the space of bounded $C^{1}\left(\mathbb{R}^{4}, \mathbb{R}^{4}\right)$-functions with bounded derivatives. If $A \in \mathscr{B}^{1}\left(\mathbb{R}^{4}, \mathbb{R}^{4}\right)$ satisfies the short range condition with respect to $X_{L}$-variable:

$$
|A(X)| \leq K\left(1+\left|X_{L}\right|\right)^{-\rho} \text { on } \mathbb{R}^{4}
$$

for some $K>0$ and some $\rho>1$, then $A$ belongs to $S(L)$.

We also say that $A$ belongs to $S$ if and only if $A$ is decomposed as

$$
A=\sum_{j=1}^{N} A_{j}, \quad A_{j} \in S\left(L_{j}\right),
$$


for some $N$ and for some subspaces $L_{j}, 1 \leq j \leq N$.

If $A$ belongs to $S$, the Dirac equation (1.1) has a unique unitary propagator $U_{A}(t, s), s, t \in \mathbb{R}$ :

$$
i \frac{d}{d t} U_{A}(t, s)=H_{A}(t) U_{A}(t, s), \quad U_{A}(s, s)=I .
$$

Moreover, we have the following.

Proposition 1.1. Let $A \in S$. Then the wave operators

$$
W_{A}^{ \pm}(s):=s-\lim _{t \rightarrow \pm \infty} U_{A}(s, t) e^{-t(t-s) H_{0}}
$$

exist for each $s \in \mathbb{R}$, where $H_{0}$ is the free Dirac operator.

$$
H_{0}=c \sum_{j=1}^{3} \alpha_{j} D_{j}+\alpha_{4} m c^{2}
$$

Remark. The free Dirac operator $H_{0}$ is a self-adjoint operator with domain $D\left(H_{0}\right)=H^{1}\left(\boldsymbol{R}^{3}, C^{4}\right)$, the Sobolev space of order 1 , and $U_{0}(t, s)=e^{-i(t-s) H_{0}}$.

The scattering operator is defined by

$$
S_{A}(s):=W_{A}^{+}(s)^{*} W_{A}^{-}(s),
$$

for each $s \in \mathbb{R}$. If some stronger condition is imposed on $A$, the scattering operator is unitary in $\mathscr{H}$. But, it is not necessarily unitary under the assumption $A \in S$.

The following useful relation follows immediately from definition:

$$
S_{A}(s)=e^{-1 s H_{0}} S_{A}(0) e^{i s H_{0}}, \quad s \in \mathbb{R} .
$$

Thanks to this relation. we know $S_{A}(s)$ for all $s \in \mathbb{R}$ if $S_{A}\left(s_{0}\right)$ is given for some $s_{0}$. The electromagnetic field $F_{A}$, determined by $A$, is defined by

$$
F_{A}=\left(F_{A}^{k^{k}}\right)_{0 \leq j<k \leq 3}=\left(\frac{\partial A^{k}}{\partial x_{j}}-\frac{\partial A^{\jmath}}{\partial x_{k}}\right)_{0 \leq j<k \leq 3}: \mathbb{R}^{4} \longrightarrow \boldsymbol{R}^{6},
$$

where $x_{0}=t$.

It should be recalled that the potential is not uniquely determined by the field and that it is not the potential but the field that can be an observable quantity. The following theorem shows that the scattering operator is determined by the field not by the potential. 
Theorem 1.2. Let $A_{(1)}$ and $A_{(2)}$ belong to $S$ and suppose that

$$
A_{(2)}-A_{(1)}=\sum_{j=1}^{N} A_{j}, \quad A_{j} \in S\left(L_{j}\right), \quad \operatorname{dim} L_{j} \geq 2, \quad 1 \leq j \leq N,
$$

and that $F_{A(1)}=F_{A(2)}$. Then $S_{A(1)}(s)=S_{A(2)}(s)$ for all $s \in \mathbb{R}$.

We next consider the inverse problem.

For a subspace $L \neq\{0\}$ of $\mathbb{R}^{4}$ the class $\widetilde{S}(\mathbb{L})$ of electromagnetic fields is defined by

$$
\widetilde{S}(L):=\left\{F \in \mathscr{B}^{0}\left(\mathbb{R}^{4}, \mathbb{R}^{6}\right) ; \int_{0}^{\infty} g_{F}^{L}(r) d r<\infty\right\},
$$

where $g_{F}^{L}(r):=\sup _{\left|X_{L}\right| \geq r}|F(X)|$ and $\mathscr{B}^{0}\left(\mathbb{R}^{4}, \mathbb{R}^{6}\right)$ is the space of bounded continuous functions from $\mathbb{R}^{4}$ to $\mathbb{R}^{6}$.

We denote by $\Xi=(\tau, \xi) \in \mathbb{R} \times \mathbb{R}^{3}$ the dual variables of $X=(t, x)$ and define an open set $D$ in $\mathbb{R}^{4}$ by

$$
D:=\left\{(\tau, \xi) \in \mathbb{R}^{4} ;|\tau|<c|\xi|\right\}
$$

We denote by $\mathscr{S}^{\prime}\left(\mathbb{R}^{4} ; \mathbb{C}^{6}\right)$ the space of $\mathbb{C}^{6}$-valued tempered distributions and by $\widehat{F} \in \mathscr{S}^{\prime}\left(\mathbb{R}^{4} ; \mathbb{C}^{6}\right)$ the Fourier transform of $F \in \mathscr{S}^{\prime}\left(\mathbb{R}^{4} ; \mathbb{C}^{6}\right)$ :

$$
\widehat{F}(\tau, \xi)=(2 \pi)^{-2} \iint e^{-\imath t \tau-\imath x \cdot \xi} \mathbb{F}(t, x) d t d x .
$$

We denote by $\left.\widehat{F}\right|_{\Omega}$ the restriction of $\widehat{F}$ to an open set $\Omega,\left.\widehat{F}\right|_{\Omega} \in \mathscr{D}^{\prime}(\Omega)$.

Theorem 1.3. Suppose that $A=\sum_{j=1}^{N} A_{3}$ with $A, \in S(L$,$) and that F_{A}=\sum_{k=1}^{N^{\prime}} F_{k}$ with $F_{k} \in \widetilde{S}\left(L_{k}^{\prime}\right)$. Then $\left.\widehat{F_{A}}\right|_{D \backslash \Sigma}$ can be reconstructed from $S_{A}(0)$, where

$$
\sum:=\left(\bigcup_{\substack{1 \leq j \leq N \\ \operatorname{dm} L L_{j}=1}} L_{j}\right) \cup\left(\bigcup_{\substack{1 \leq k \leq N \\ \operatorname{dim} L_{k}^{\prime}=1}} \mathbb{L}_{k}^{\prime}\right)
$$

Remark. The decompositions of $A$ and $F_{A}$ are not unique and the exceptional set $\sum$ depends on the decompositions. Let $\mathscr{A}$ be the set of all pairs of decompositions of $A$ and $\mathbb{F}_{A}$, and denote by $\sum_{a}$ the $\sum$ in (1.4) for $a \in \mathscr{A}$. Then $\Sigma$ in Theorem 1.3 may be replaced by the smaller set

$$
\tilde{\Sigma}:=\bigcap_{a \in \mathscr{A}} \sum_{a}
$$

Theorem 1.3 tells us nothing about $\widehat{F}_{A}$ on $D^{c}$, the complement of $D$. If $\widehat{F}_{A}$ 
on $D^{c}$ does not contributes to the scattering operator, this result would be satisfactory. However, the following Proposition shows that this is not the case.

Proposition 1.4 Let $c=m=1$ and let

$$
D_{1}:=\left\{(\tau, \xi) \in \mathbb{R} \times \mathbb{R}^{3} ; \tau>\sqrt{|\xi|^{2}+4}\right\} .
$$

Then for any $\phi \in \&\left(\mathbb{R}_{t} \times \mathbb{R}_{x}^{3} ; \mathbb{R}_{\mathbb{R}}\right)$ such that supp $\widehat{\phi} \cap D_{1} \neq \emptyset$, the scattering operator $S_{\lambda A}(0)$ for $(1.1)$ with the potential $\lambda A=(\lambda \phi, 0,0,0)$ satisfies $S_{\lambda A}(0) \neq I$ for any small $\lambda \neq 0$.

Remark. The author does not know whether the field $F_{A}$ can be reconstructed completely from the scattering operator if the support of $\widehat{F_{A}}$ has an intersection with $D^{c}$, while on the other hand an affirmative answer is known in the case of Schrödinger equations [We1]. The velocity of a relativistic particle cannot exceed that of light, whereas a nonrelativistic particle can have any speed. This is one of the greatest differences between a particle obeying a Dirac equation and one obeying a Schrödinger equation. The following intuitive argument shows that the field $F$ with supp $\widehat{F} \subset D^{\prime}:=\{(\tau, \xi) ;|\tau|>c|\xi|\}$ may be peculiar one from the point of view of the relativity. We write formaly

$$
F(t, x)=(2 \pi)^{-2} \int_{D^{\prime}} F_{\tau, \xi}(t, x) d \tau d \xi
$$

where $F_{\tau, \xi}(t, x):=e^{t t \tau+\imath x \cdot \xi} \widehat{F}(\tau, \xi)$ satisfies the wave equation

$$
\frac{1}{\tau^{2}|\xi|^{-2}} \frac{\partial^{2}}{\partial t^{2}} F_{\tau, \xi}(t, x)=\Delta_{x} F_{\tau, \xi}(t, x)
$$

This implies that each component $F_{\tau, \xi}$ of the field $F$ propagates with velocity $|\tau||\xi|^{-1}>c$, which contradicts the relativity.

We can determine the field completely, if we impose some conditions on the field, as corollaries of Theorem 1.3.

Theorem 1.5. Suppose ( $i$ ) and (ii) in Theorem 1.3. Moreover, we assume $\Sigma$ $\cap D=\emptyset$ and

$$
\left(\operatorname{supp} \widehat{F_{A}} \backslash\{0\}\right) \cap D^{c}=\emptyset .
$$

Then $F_{A}$ can be completely reconstructed from $S_{A}(0)$.

Using this theorem we can treat time-independent potentials. 
Corollary 1.6. Suppose ( $i$ ) and (ii) in Theorem 1.3 with $\Sigma \cap D=\emptyset$. Moreover, suppose that for each $k=1, \cdots, N^{\prime}$ there exists $V_{k} \in T:=\left\{X=(t, x) \in \mathbb{R}^{4}\right.$; $c|t|>|x|\}$ such that

$$
F_{k}\left(s V_{k}+X\right)=F_{k}(X), \quad s \in \mathbb{R}, \quad X \in \mathbb{R}^{4} .
$$

Then $F_{A}$ can be reconstructed completely from $S_{A}(0)$.

Remark 1. In Section 5 we will show that each $F_{k}$ satisfying (1.6) may be made time-independent if a suitable inertial frame is chosen.

Remark 2. If $A \in S$ is independent of $t$ and satisfies

$$
|A(x)|+\left|F_{A}(x)\right| \leq \mathbb{K}(\mathbb{1}+|x|)^{-\rho} \text { on } \mathbb{R}^{3},
$$

for some constants $K>0$ and $\rho>\mathbb{1}$. Then Corollary 1.6 shows that $F_{A}(x)$ can be completely reconstructed from the scattering operator $S_{A}(0)$. This has been known under different conditions in [It], [J] and [Is]. Roughly speaking, the decay rate of the potential is supposed to be $\rho>3$ in [It], $\rho>3 / 2$ in [J] and $\rho>$ 2 in [Is]. However, the decay condition on the field is not imposed in [J], and the magnetic field is not treated in [Is].

The next theorem shows that the field is uniquely determined by the scattering operator under some exponential decay condition.

Theorem 1.7. Let $A \in S$.

(1) Suppose there exists $V \in S^{3}, S^{3}$ being the unit sphere in $\mathbb{R}^{4}$, such that

$$
\left|F_{A}(X)\right| \leq K e^{-\delta\left|\langle V, X\rangle R^{4}\right|} \text { on } \mathbb{R}^{4},
$$

for some constants $K>0$ and $\delta>0$.

Then $\left.\widehat{F_{A}}\right|_{R^{\prime} \backslash L}$ is determined by $S_{A}(0)$, where $L$ is the one-dimensional subspace spanned by $V$.

(2) Suppose, in addition to the assumption of (1), that there exist $V^{\prime} \in S^{3}$ not parallel to $V$ and a bounded function $g$ satisfying $g(t)(1+t)^{-1} \in \mathbb{L}^{1}((0, \infty))$ such that

$$
\left|F_{A}(X)\right| \leq g\left(\left|\left\langle V^{\prime}, X\right\rangle_{R^{4}}\right|\right) e^{-\delta \mid\langle V, X\rangle_{R^{4}}} \text { on } \mathbb{R}^{4}
$$

Then $F_{A}$ is uniquely determined by $S_{A}(0)$.

Remark. Roughly speaking, (2) implies that if $F_{A}$ satisfies

$$
\left|F_{A}(X)\right| \leq K\left(1+\left|\left\langle V_{1}, X\right\rangle_{R^{1}}\right|\right)^{-\rho} e^{-\delta\left|\left\langle V_{2}, X\right\rangle R^{4}\right|} \text { on } \mathbb{R}^{4}
$$

for some linearly independent $V_{1}, V_{2} \in \mathbb{R}^{4} \cap S^{3}$ and for some $K>0, \rho>0$ and $\delta>$ 0 , then $F_{A}$ is completely determined by the scattering operator. 
It is an important problem in physics as well as in mathematics to know whether the external field can be determined from the scattering operator. In the case of Schrödinger operators with time-independent potentials, it is known, since Faddeev $[\mathrm{F}]$, that the potential can be reconstructed from the high-energy behavior of the scattering matrices (see, for example, [Is-K], [Ne], [Sa], [Wa], $[\mathrm{Ni}]$ ). The proofs are based on a stationary representation of the scattering matrices and some resolvent estimates at the high-energy range. Using a similar stationary method, the author [It] has proved that the electromagnetic fileld can be reconstructed from the high-energy behavior of the scattering matrices of the Dirac operator with a time-independent potential. In [Is] Isozaki has obtained a similar result as well as an uniqueness result for the fixed energy problem by a different method.

On the other hand, Enss and Weder [E-We] have found a new method, a time-dependent method (a geometric method), to reconstruct the potential from the high-energy asymptotics of the scattering operator in the case of Schrödinger operators without magnetic fields. Since their method is simple, it can be applicable to many cases. Recently, Arians [A1] has applied their method to reconstruct the electromagnetic field for the Schrödinger operator with a time-independent electromagnetic potential. See also [A2, We2, We3]. On the other hand, Weder [We1] has shown that the potential can be completely reconstructed from the scattering operator for the Schrödinger equations with a time-dependent potential. For Dirac operators with a time-independent potential Jung [J] has reconstructed the electromagnetic field by using the geometric method. Some of his proofs are applicable to the case of time-dependent potentials (Proposition 2.2)

At the end of this section we describe the plan of our paper. Section 2 contains the proofs of Proposition 1.1, Theorems 1.2 and 1.3. Proposition 1.1 is proved by Cook's method with the help of estimates of the propagator for the free Dirac operator in the classically forbidden region. The proof of Theorem 1.2 is carried out by constructing a function $u_{1}$ satisfying $\nabla_{X} u_{1}=A$ and decaying in some directions. The proof of Theorem 1.3 is based on Proposition 2.2 , which gives high-energy asymptotics of the scattering operator. Proposition 2.2 is proved by using a time-dependent method, due to Enss and Weder, as in [J]. By means of Proposition 2.2 and Stoke's theorem we can prove that some line integrals (2.31) for the electromagnetic field are constructed from the scattering operator. If the magnetic field does not exist, the line integrals are the same ones as appeared in [St] and [R-S], in which the inverse scattering for wave equations with time-dependent potentials is studied. Roughly speaking, Theorem 1.3 is proved by constructing the field from the line integrals with the help of the Fourier transform. The proofs of Theorems 1.5 and 1.7, based on Theorem 1.3, are given in Section 3. Proposition 1.4 is proved by investigating the second term of the Dyson expansion of the scattering operator in Section 4. 
Dirac equations are relativistic invariant. In Section 5 we will show that our assumptions and results are independent of the choice of the inertial frame.

\section{§ 2. Proofs of Proposition 1.1, Theorems 1.2 and 1.3}

In this section we will give proofs of Proposition 1.1, Theorem 1.2 and 1.3.

Proof of Proposition 1.1. Let $A=\sum_{j=1}^{N} A_{j}, A_{j} \in S\left(L_{j}\right)$ and define

$$
K_{A}:=\bigcup_{j=1}^{N}\left\{\xi \in \mathbb{R}^{3} ; V^{+}(\xi) \in L_{j}^{+} \text {or } V^{-}(\xi) \in \mathbb{L}_{j}^{+}\right\}
$$

where

$$
V^{ \pm}(\xi):=(1, \pm v(\xi))=\left(1, \pm c^{2} \xi / \sqrt{c^{2}|\xi|^{2}+m^{2} c^{4}}\right)
$$

and $L^{\perp}$ is the orthogonal complement of $L \subset \mathbb{R}^{4}$. Since $K_{A}$ is a closed null set in $\mathbb{R}^{3}$,

$$
G:=\left\{f \in \mathscr{S}\left(\mathbb{R}^{3}, \mathbb{C}^{4}\right) ; \widehat{f} \in C_{0}^{\infty}\left(\mathbb{R}^{3} \backslash\left(K_{A} \cup\{0\}\right) ; \mathbb{C}^{4}\right)\right\}
$$

is dense in $\mathscr{H}$, where $\widehat{f}$ is the Fourier transform of $f$. By Cook's method, Proposition 1.1 follows from it:

$$
\int_{-\infty}^{\infty}\left\|A_{1}(t, \cdot) e^{-\imath t H_{0}} P_{ \pm} f\right\| d t<+\infty, \quad j=1, \cdots N,
$$

for all $f \in G$, where $P_{ \pm}$is the orthogonal projection in $\mathscr{H}$ onto the positive/negative energy subspace of $H_{0}$,

$$
P_{ \pm}:=\frac{1}{2}\left(I \pm \frac{H_{0}}{\left|H_{0}\right|}\right)
$$

and $\|\cdot\|$ denotes the norm of $\mathscr{H}$.

We have for $\delta>0$

$$
\begin{aligned}
& \left\|A_{,}(t, \cdot) e^{-\imath t H_{0}} P_{ \pm} f\right\| \\
& \leq\left\|A_{,}(t, \cdot) \chi\left(\left|(t, x)_{L},\right| \geq \delta|t|\right)\right\|_{L^{\infty}}\|f\| \\
& +\left\|A_{j}(t, \cdot)\right\|_{L^{\infty}}\left\|\chi\left(\left|(t, x)_{L},\right| \leq \delta|t|\right) e^{-\imath t H_{0}} P_{ \pm} f\right\|,
\end{aligned}
$$

where $\chi(M(x))$ denotes the multiplication operator by the characteristic function of $x$ satisfying the condition $M(x)$ and $(t, x)_{L}$ is the orthogonal projection of $(t, x) \in \mathbb{R}^{4}$ onto $L$. Since $A_{j} \in S\left(L_{j}\right)$, the first term on the right is bounded by $g_{A}^{L},(\delta|t|)\|f\|$ and so is integrable with respect to $t \in \mathbb{R}$. Therefore it suffices to show that there exists a small $\delta>0$ such that 


$$
\left\|\chi\left(\left|(t, x)_{L}\right| \leq \delta|t|\right) e^{-i t H_{0}} P_{ \pm} f\right\| \leq K_{n}(1+|t|)^{-n}
$$

for any $n>0$, where $K_{n}$ is a constant independent of $t$. The symbol of $H_{0}$.

$$
h_{0}(\xi):=c \sum_{j=1}^{3} \alpha_{j} \xi_{j}+\alpha_{4} m c^{2},
$$

has eigenvalues $\pm \sqrt{c^{2}|\xi|^{2}+m^{2} c^{4}}$ and the associated eigenprojection is given by

$$
P^{ \pm}(\xi)=\frac{1}{2}\left(I_{4} \pm \frac{h_{0}(\xi)}{\sqrt{c^{2}|\xi|^{2}+m^{2} c^{4}}}\right)
$$

Hence we have

$$
\left(e^{-i t H_{0}} P_{ \pm} f\right)(x)=(2 \pi)^{-3 / 2} \int e^{i g \pm(t, x, \xi)} \widehat{f}_{ \pm}(\xi) d \xi
$$

where $g^{ \pm}(t, x, \xi)=\langle x, \xi\rangle \mp t \sqrt{c^{2}|\xi|^{2}+m^{2} c^{4}}$ and $\widehat{f}_{ \pm}(\xi)=P^{ \pm}(\xi) \widehat{f}(\xi)$.

We claim that there exists a constant $K>0$ such that

$$
\left|\nabla_{\xi} g^{ \pm}(t, x, \xi)\right| \geq K(|t|+|x|)
$$

for all $(t, x, \xi) \in \Omega_{j} \times \operatorname{supp} \widehat{f}$ if $\delta>0$ is sufficiently small, where

$$
\Omega_{j}:=\left\{(t, x) \in \mathbb{R} \times \mathbb{R}^{3} ;|t|>1,\left|(t, x)_{L}\right| \leq \delta|t|\right\} .
$$

Since $|v(\xi)| \leq c$ and

$$
\nabla_{\xi} g^{ \pm}(t, x, \xi)=x \mp t v(\xi)
$$

it follows that

$$
\left|\nabla_{\xi} g^{ \pm}\right| \geq|x|-c|t| .
$$

On the other hand, for $(t, x, \xi) \in \Omega, \times \operatorname{supp} \widehat{f}$, we have

$$
\begin{aligned}
|x \mp t v(\xi)| & =\left|(t, x)-t V^{ \pm}(\xi)\right| \geq\left|(t, x)_{L}-t V^{ \pm}(\xi)_{L}\right| \\
& \geq|t|\left(\left|V^{ \pm}(\xi)_{L}\right|-\delta\right) \geq\left(\delta_{1}-\delta\right)|t|,
\end{aligned}
$$

where $\delta_{1}:=\inf _{\xi \in \operatorname{supp} f}\left|V^{ \pm}(\xi)_{L}\right|>0$, since dist $\left(K_{A}\right.$, supp $\left.\widehat{f}\right)>0$. Thus, if $0<\delta<\delta_{1}$, taking account of (2.6), we obtain (2.4). Therefore, it is easy to see that, on $\Omega_{j}$ $\times \operatorname{supp} \widehat{f}, \nabla_{\xi} g^{ \pm} /\left|\nabla_{\xi} g^{ \pm}\right|^{2} \in C^{\infty}$ and

$$
\left|\partial_{\xi}^{r}\left(\frac{\nabla_{\xi} g^{ \pm}}{\mid \nabla_{\xi} g^{ \pm 2}}\right)\right| \leq K_{r}(|t|+|x|)^{-1}
$$

for any $\gamma$. Thus, using integration by parts together with

$$
i e^{\imath g^{ \pm}}=\frac{\nabla_{\xi}^{ \pm} g}{\left|\nabla_{\xi} g^{ \pm}\right|^{2}} \cdot \nabla_{\xi} e^{\imath g^{ \pm}}
$$


we conclude that for any $n$ there exists $K_{n}$ such that

$$
\left|\left(e^{-\imath t H_{0}} P_{ \pm} f\right)(x)\right| \leq K_{n}(1+|t|+|x|)^{-n},
$$

for all $(t, x) \in \Omega_{j}$. This implies (2.3) and completes the proof of Proposition 1.1 .

Remark. If $\mathbb{L}$ is a nontrivial subspace of $\mathbb{R}^{4}$ and if $J(\sigma)$ is a monotone decreasing function on $(0, \infty)$ with $\lim _{\sigma \rightarrow \infty} J(\sigma)=0$, then

$$
s-\lim _{t \rightarrow \pm \infty} J\left(\left|(t, \cdot)_{L}\right|\right) e^{-\imath t H_{0}}=0 .
$$

This can be proved in the same way as above by replacing $A_{j}(t, x)$ by $\mathbb{J}\left(\left|(t, x)_{L}\right|\right)$. This fact will be used in the proof of Lemma 2.1 below.

Proof of Theorem 1.2. Let $A:=A_{(2)}-A_{(1)}$. Since $F_{A}=0$,

$$
u(X):=\int_{0}^{1}\langle X, A(s X)\rangle_{R^{\star}} d s
$$

is in $C^{1}\left(\mathbb{R}^{4}\right)$ and satisfies

$$
\nabla_{X} u=A \text { on } \mathbb{R}^{4}
$$

Let $V \in S^{3} \backslash\left(\cup_{j=1}^{N} L_{j}^{\perp}\right)$, where $S^{3}$ is the unit sphere in $\mathbb{R}^{4}$. Then $V_{L} \neq 0$ for $j=\mathbb{1}$, $\cdots, N$. Using (2.10), we have for $0<r_{1}<r_{2}$

$$
\begin{aligned}
\left|u\left(r_{2} V\right)-u\left(r_{1} V\right)\right| & =\left|\int_{r_{1}}^{r_{2}} \frac{d}{d r} u(r V) d r\right| \\
& \leq \int_{r_{1}}^{r_{2}}\left|\langle V, A(r V)\rangle_{R^{4}}\right| d r \\
& \leq \sum_{j=1}^{N} \int_{r_{1}}^{r_{2}} g_{A_{j}}^{L_{s}}\left(r\left|V_{L j}\right|\right) d r
\end{aligned}
$$

(2.11) implies that the limit

$$
u_{\infty}(\mathbb{V}):=\lim _{r \rightarrow+\infty} u(r \mathbb{V})
$$

exists. We next prove that the limit $u_{\infty}(V)$ is independent of $V \in S^{3} \backslash\left(\cup_{j=1}^{N} L_{j}^{+}\right)$. Let $V, V^{\prime} \in S^{3} \backslash\left(\cup_{j=1}^{N} L_{j}^{\perp}\right)$. Since $\operatorname{dim} L_{j}^{\perp} \leq 2, S^{3} \backslash\left(\cup_{j=1}^{N} L_{j}^{\frac{1}{j}}\right)$ is connected. Hence, we may prove $u_{\infty}(V)=u_{\infty}\left(V^{\prime}\right)$ for $V, V^{\prime}$ such that the segment between $V$ and $V^{\prime}$ has no intersection with $\cup_{j=1}^{N} \mathbb{L}_{j}^{+}$. Then there exists $\varepsilon_{0}>0$ such that

$$
\inf _{\sigma \in[0,1]}\left|\sigma\left(V_{L}^{\prime}-V_{L}\right)+V_{L}\right|>\varepsilon_{0}, \quad j=1, \cdots, N .
$$

Using (2.10) again, we estimate 


$$
\begin{aligned}
\left|u(r V)-u\left(r V^{\prime}\right)\right| & \leq \int_{0}^{1}\left|r\left(V^{\prime}-V\right)\right|\left|A\left(r\left(\sigma\left(V^{\prime}-V\right)+V\right)\right)\right| d \sigma \\
& \leq 2 \sum_{j=1}^{N} r g_{A}^{L,}\left(\varepsilon_{0} r\right) .
\end{aligned}
$$

Since $g_{A,}^{L,}(r)$ is monotone decreasing and integrable,

$$
r g(r) \leq 2 \int_{r / 2}^{r} g(s) d s \rightarrow 0
$$

as $r \rightarrow \infty$, and we obtain $u_{\infty}\left(V^{\prime}\right)=u_{\infty}(V) \equiv u_{\infty}$. Accepting the following lemma, we proceed our proof of Theorem 1.2.

Lemma 2.1. Let $u_{1}(X)=u(X)-u_{\infty}$. Then

$$
s-\lim _{t \rightarrow \pm \infty}\left(e^{i u_{1}(t, x)}-1\right) e^{-\imath t H_{0}} f=0
$$

for all $f \in \mathscr{H}$.

Now we define a unitary propagator

$$
\widetilde{U}(t, s):=e^{-\imath u_{1}(t, x)} U_{A(2)}(t, s) e^{i u_{1}(s, x)} .
$$

Then, taking account of (2.10), we can easily verify that

$$
i \frac{d}{d t} \widetilde{U}(t, s)=H_{A(1)}(t) \widetilde{U}(t, s), \quad \widetilde{U}(s, s)=I .
$$

This means $\widetilde{U}=U_{A(1)}$ by the uniqueness of the propagator. Hence, in virtue of Lemma 2.1,

$$
\begin{aligned}
W_{A(1)}^{ \pm}(s) & =s-\lim _{t \rightarrow \pm \infty} e^{-\imath u_{1}(s, x)} U_{A(2)}(s, t)\left\{\left(e^{i u_{1}(t, x)}-1\right)+1\right\} e^{-t(t-s) H_{0}} \\
& =e^{-\imath u_{1}(s, x)} W_{A_{(2)}}^{ \pm}(s)
\end{aligned}
$$

Thus we have proved $S_{A(1)}(s)=S_{A(2)}(s)$.

Proof of Lemma 2.1. We use the same notation as in the proof of Proposition 1.1. It suffices to show (2.12) for a fixed $f \in G$. Since supp $\widehat{f} \cap K_{A}$ $=\emptyset$, we can find $\varepsilon_{0} \in(0,1 / 2)$ such that

$$
\left|V^{ \pm}(\xi)_{L, \downarrow} \leq\left(1-2 \varepsilon_{0}\right)\right| V^{ \pm}(\xi) \mid,
$$

for $\xi \in \operatorname{supp} \widehat{f}, j=1, \cdots, N$. Define

$$
\Gamma_{j}:=\left\{X \in \mathbb{R}^{4} ;\left|X_{L}\right| \leq \delta_{0}|X|\right\}
$$

for $j=1, \cdots, N$, where $\delta_{0}$ is a fixed constant with $0<\delta_{0} \leq \varepsilon_{0}$. Then 


$$
\begin{aligned}
\left|\left\langle X, V^{ \pm}(\xi)\right\rangle\right| & \leq\left|X_{L^{\prime}}\left\|V^{ \pm}(\xi)_{L_{L}}|+| X_{L}\right\| V^{ \pm}(\xi)_{L_{t}}\right| \\
& \leq\left(1-\varepsilon_{0}\right)|X|\left|V^{ \pm}(\xi)\right|
\end{aligned}
$$

if $\xi \in \operatorname{supp} \widehat{f}$ and $X=(t, x) \in \Gamma_{j}$. Thus,

$$
\begin{aligned}
\left|\nabla_{\xi} g^{ \pm}(t, x, \xi)\right| & =\left|X-t V^{ \pm}(\xi)\right| \\
& =|X|^{2}+|t|^{2}\left|V^{ \pm}(\xi)\right|^{2}-2 t\left\langle X, V^{ \pm}(\xi)\right\rangle \\
& \geq \varepsilon_{0}\left(|X|^{2}+|t|^{2}\left|V^{ \pm}(\xi)\right|^{2}\right) \\
& \geq \varepsilon_{0}\left(|X|^{2}+|t|^{2}\right) .
\end{aligned}
$$

Thus, due to the same argument as in the proof of (2.8), it follows that for any $n$ there exists $K_{n}$ such that

$$
\left|\left(e^{-\imath t H_{0}} f\right)(x)\right| \leq K_{n}(1+|t|+|x|)^{-n} .
$$

if $|t| \geq 1$ and $(t, x) \in \Gamma$, for some $j$. Now

$$
\begin{aligned}
\left(e^{i u_{1}(t, x)}-1\right)\left(e^{-\imath t H_{0}} f\right)(x) \\
\quad=\left(e^{i u_{1}(t, x)}-1\right) \prod_{j=1}^{N}\left(\chi\left((t, x) \notin \Gamma_{j}\right)+\chi\left((t, x) \in \Gamma_{j}\right)\right)\left(e^{-t t H_{0}} f\right)(x) \\
\quad=\left(e^{i u_{1}(t, x)}-1\right) \cdot \prod_{j=1}^{N} \chi\left((t, x) \notin \Gamma_{j}\right) \cdot\left(e^{-t t H_{0}} f\right)(x) \\
\quad+\left(e^{i u_{1}(t, x)}-1\right)\left[\sum_{j=1}^{N} \tilde{\chi},(t, x) \chi\left((t, x) \in \Gamma_{j}\right)\right]\left(e^{-\imath t H_{0}} f\right)(x) \\
\quad \equiv I_{1}(t, x)+I_{2}(t, x),
\end{aligned}
$$

where $\tilde{\chi}_{j}(t, x)$ are products of characteristic functions. In view of (2.14) we have

$$
s-\lim _{t \rightarrow \pm \infty} I_{2}(t, \cdot)=0 .
$$

Let $X \notin \cup{ }_{j=1}^{N} L_{j}^{\perp}$. Then

$$
\begin{aligned}
u_{\infty} & =\lim _{r \rightarrow \infty} u(r X) \\
& =\lim _{r \rightarrow \infty} \int_{0}^{1}\langle r X, A(\tau \gamma X)\rangle d \tau \\
& =\int_{0}^{\infty}\langle X, A(\tau X)\rangle d \tau,
\end{aligned}
$$

and so

$$
u_{1}(X)=\int_{\infty}^{1}\langle X, A(\tau X)\rangle d \tau=\sum_{j=1}^{N} \int_{\infty}^{1}\left\langle X, A_{j}(\tau X)\right\rangle d \tau .
$$

Thus, noting that $(t, x) \notin \bigcup_{j=1}^{N} L_{j}^{\perp}$ if $(t, x) \notin \bigcup_{j=1}^{N} \Gamma_{j}$, we estimate 


$$
\begin{aligned}
\left|e^{i u_{1}(t, x)}-1\right| & \leq\left|u_{1}(t, x)\right| \\
& \leq \sum_{j=1}^{N} \int_{1}^{\infty}|(t, x)|\left|A_{j}(s(t, x))\right| d s \\
& \leq \sum_{j=1}^{N} \int_{1}^{\infty} \delta_{0}^{-1}\left|(t, x)_{L,}\right| g_{A^{\prime}}^{L^{\prime}}\left(s\left|(t, x)_{L j}\right|\right) d s \\
& \leq \sum_{j=1}^{N} \int_{\left|(t, x)_{L}\right|}^{\infty} \delta_{0}^{-1} g_{A^{\prime}}^{L^{\prime}}(s) d s
\end{aligned}
$$

for $(t, x) \notin \cup_{j=1}^{N} \Gamma_{j}$. Hence, taking account of $(2.9)$ and $g_{A}^{L,} \in L^{1}((0, \infty))$, we obtain

$$
s-\lim _{t \rightarrow \pm \infty} I_{1}(t, \cdot)=0
$$

and finish the proof of Lemma 2.1.

Remark. Let $\phi$ be a real-valued function on $\mathbb{R}_{t}$ with

$$
|\phi(t)| \leq K(1+|t|)^{-1-\varepsilon} \text { uniformly on } \mathbb{R}_{t}
$$

for some $K>0$ and $\varepsilon>0$, and let $A=(\phi, 0)$. Then we see that $F_{A}=F_{0}=0$ and

$$
U_{A}(t, s)=e^{-\imath(\Phi(t)-\Phi(s))} e^{-\imath(t-s) H_{0}}, \text { where } \Phi(t)=\int_{-\infty}^{t} \phi(s) d s
$$

Thus it follows that

$$
W_{A}^{ \pm}(s)=e^{\imath L_{\infty}^{ \pm \infty} \phi(t) d t-\imath \Phi(s)}
$$

and so

$$
S_{A}(s)=e^{-i \int_{-\infty}^{\infty} \phi(t) d t}
$$

This means that $S_{A}(0)=S_{0}(0)=I$ if and only if $\int_{-\infty}^{\infty} \phi(t) d t \in 2 \pi \mathbb{Z}$.

The above argument shows that Theorem 1.2 does not hold in general if the condition $\operatorname{dim} L, \geq 2$ is dropped out.

We next prove Theorem 1.3. To do so, we have to prepare some notations.

For a subspace $L$ we define a set $C(L) \subset S^{2}$ by

$$
C(L):=\left\{\omega \in S^{2} ; \widetilde{\omega} \in L^{\perp}\right\},
$$

where $\widetilde{\omega}:=(1, c \omega) \in \mathbb{R}^{4}$. We sometimes write $C(V)=C(L)$ if $L$ is the onedimensional subspace spanned by $V \neq 0 \in \boldsymbol{R}^{4}$. If $V=\left(v_{0}, v\right) \in \boldsymbol{R} \times \boldsymbol{R}^{3}$, 


$$
C(\mathbb{V})=\left\{\omega \in S^{2} ;\langle v, \omega\rangle_{R^{3}}=-v_{0} / c\right\} .
$$

Hence, $C(V)$ is a circle on $S^{2}$ if $V \in D$, one point $\left\{-v_{0} v / c|v|^{2}\right\}$ on $S^{2}$ if $V \in \bar{D} \backslash D$ and empty if $V \notin \bar{D}$. Moreover, it is easy to see that

$$
C\left(V_{1}\right)=C\left(V_{2}\right) \neq \emptyset \Longleftrightarrow V_{1} / / V_{2} \text {. }
$$

On the other hand,

$$
C(\mathbb{L})=\bigcap_{j=1}^{N} C\left(V_{j}\right)
$$

if $\left\{V_{1}, \cdots, V_{N}\right\}$ is a basis of $\mathbb{L}$. Therefore, the number of elements of $C(\mathbb{L})$ is at most two if $\operatorname{dim} \mathbb{L}=2$. Moreover, it is at most one and zero if $\operatorname{dim} L=3$ and $\operatorname{dim} \mathbb{L}$ $=4$, respectively, since $\operatorname{dim} L^{\perp}=1$ and $\operatorname{dim} \mathbb{L}^{\perp}=0$, respectively.

For $A=\sum_{j=1}^{N} A_{j} \in S$ with $A_{j} \in S\left(L_{j}\right)$ we define

$$
C_{A}:=\bigcup_{j=1}^{N} C\left(\mathbb{L}_{j}\right)
$$

Then line integrals

$$
K_{A}^{\omega}(\eta):=\int_{-\infty}^{\infty}\langle\widetilde{\omega}, A(t \widetilde{\omega}+\eta)\rangle_{R^{4}} d t . \quad \eta \in \mathbb{R}_{t} \times \mathbb{R}_{x}^{3}
$$

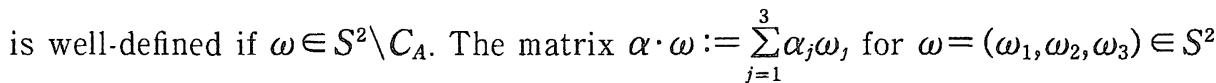
has eigenvalues 1 and -1 with multiplicity two, respectively. Thus, $P_{ \pm}(\omega):=$ $\frac{1}{2}(1 \pm \alpha \cdot \omega)$ is the eigenprojection associated with the eigenvalue \pm 1 of $\alpha \cdot \omega$.

The proof of Theorem 1.3 is based on the following proposition.

Proposition 2.2. Let $A \in S$ and $s \in \mathbb{R}$. Then

$$
w-\lim _{E \rightarrow+\infty} e^{-\imath E \omega \cdot x} P_{ \pm} S_{A}(s) P_{ \pm} e^{\imath E \omega \cdot x}=e^{i K_{1}^{\ddagger \omega}(s, x)} P_{ \pm}(\omega)
$$

if $\pm \omega \in S^{2} \backslash C_{A}$. Here, $e^{ \pm I E \omega \cdot x}$ and $e^{\imath K_{A}^{ \pm \omega}(s, x)}$ are multiplication operators.

Remark 1 . We can also show that

$$
w-\lim _{E \rightarrow+\infty} e^{-i E \omega \cdot x} P_{ \pm} S_{A}(s) P_{\mp} e^{\imath E \omega \cdot x}=0
$$

for each $s \in \mathbb{R}$ if both $\omega$ and $-\omega$ belong to $S^{2} \backslash C_{A}$.

Remark 2. If the potential $A$ is time-independent and short-range, 
Proposition 2.2 has been already proved by Jung [J], and his proof is applicable to our case with a slight modification.

Lemma 2.3. Let $f \in \mathscr{H}$.

(i) Suppose $\omega \in S^{2} \backslash C_{A}$. Then there exists $E_{0}>0$ such that

$$
\lim _{t \rightarrow \pm \infty}\left\|\left(W_{A}^{ \pm}(0)-U_{A}(0, t) e^{-t t H_{0}}\right) P_{+} e^{i E \omega \cdot x} f\right\|=0
$$

uniformly in $E \geq E_{0}$.

(ii) Suppose $-\omega \in S^{2} \backslash C_{A}$. Then there exists $E_{0}>0$ such that

$$
\lim _{t \rightarrow \pm \infty}\left\|\left(W_{A}^{ \pm}(0)-U_{A}(0, t) e^{-\imath t H_{0}}\right) P_{-} e^{\imath E \omega \cdot x} f\right\|=0
$$

uniformly in $E \geq E_{0}$.

The proof is very similar to that of Proposition 1.1 .

Proof. We use the notation in the proof of Proposition 1.1 and prove (i) for $f \in G$, since $G$ is dense in $\mathscr{H}$. It suffices to show the following estimate (see (2.3)):

$$
\left|\left(e^{-\imath t H_{0}} P_{+} e^{\imath E \omega \cdot x} f\right)(x)\right| \leq C_{N}(1+|t|+|x|)^{-N}
$$

on $\Omega_{j}$ uniformly in $E \geq E_{0}$. We write

$$
\begin{aligned}
\left(e^{-\imath t H_{0}} P_{+} e^{\imath E \omega \cdot x} f\right)(x) & =(2 \pi)^{-3 / 2} \int e^{\imath g^{+}(t, x, \xi)} P^{+}(\xi) \widehat{f}(\xi-E \omega) d \xi \\
& =(2 \pi)^{-3 / 2} \int e^{\imath g^{+}(t, x, \xi+E \omega)} P^{+}(\xi+E \omega) \widehat{f}(\xi) d \xi .
\end{aligned}
$$

Since $\widetilde{\omega}_{L} \neq 0$ for $j=1, \cdots, N$ and $\lim _{E \rightarrow+\infty} V^{+}(\xi+E \omega)=\widetilde{\omega}$ uniformly in $\xi \in \operatorname{supp} \widehat{f}$, we can find $E_{0}>0$ and $\delta_{1}^{\prime}>0$ such that

$$
\left|V^{+}(\xi+E \omega)_{L_{j}}\right| \geq \delta_{1}^{\prime}, \quad j=1, \cdots, N,
$$

for any $E \geq E_{0}$ and $\xi \in \operatorname{supp} \widehat{f}$. Moreover, we can verify that $\left|\partial_{\xi}^{r} P^{+}(\xi+E \omega)\right|$ is uniformly bounded in $\xi \in \operatorname{supp} \widehat{f}$ and in $E>E_{0}$ for each $\gamma$. Thus we can prove (2.22) in the same way as in the proof of (2.8) by using $\delta_{1}^{\prime}$ in place of $\delta_{1}$.

Lemma 2.4. Let $f, g \in \mathscr{H}$ and let $\pm \omega \in S^{2} \backslash C_{A}$. Then

$$
\begin{aligned}
& \lim _{t \rightarrow+\infty}\left(e^{-\imath E \omega \cdot x} P_{ \pm} e^{\imath t H_{0}} U_{A}(t,-t) e^{\imath t H_{0}} P_{ \pm} e^{\imath E \omega \cdot x} f, g\right) \\
& =\left(e^{-\imath E \omega \cdot x} P_{ \pm} S_{A}(0) P_{ \pm} e^{\imath E \omega \cdot x} f, g\right)
\end{aligned}
$$

uniformly in $E \geq E_{0}$. 
Proof. Denoting $W(t):=U_{A}(0, t) e^{-\imath t H_{0}}$, we have

$$
\begin{aligned}
& \mid\left(e^{-i E \omega \cdot x} P_{ \pm} W(t) * W(-t) P_{ \pm} e^{i E \omega \cdot x} f, g\right) \\
& \quad-\left(e^{-i E \omega \cdot x} P_{ \pm} S_{A}(0) P_{ \pm} e^{i E \omega \cdot x} f, g\right) \mid \\
& \quad \leq\left|\left(W(-t) P_{ \pm} e^{i E \omega \cdot x} f,\left(W(t)-W_{A}^{+}(0)\right) \mathbb{P}_{ \pm} e^{i E \omega \cdot x} g\right)\right| \\
& \quad+\left|\left(\left(W(-t)-W_{A}^{-}(0)\right) P_{ \pm} e^{i E \omega \cdot x} f, W_{A}^{+}(0) P_{ \pm} e^{i E \omega \cdot x} g\right)\right| .
\end{aligned}
$$

Hence, using Lemma 2.3, we get the desired result.

We define

$$
\begin{gathered}
Q(t, x):=H_{A}(t)-H_{0}=-c \sum_{j=1}^{3} \alpha_{j} A^{j}(t, x)-A^{0}(t, x) I_{4} \\
W_{\omega}(t, x):=\langle\widetilde{\omega}, A(t, x+c t \omega)\rangle_{R^{4}} P_{+}(\omega)+\left\langle(\widetilde{(-\omega)}, A(t, x-c t \omega)\rangle_{R^{4}} P_{-}(\omega)\right.
\end{gathered}
$$

Lemma 2.5. For each $t>0$ and each $\omega \in S^{2}$, one has

$$
s-\lim _{E \rightarrow+\infty} e^{-t E \omega \cdot x} e^{i t H_{0}} U_{A}(t,-t) e^{\imath t H_{0}} e^{\imath E \omega \cdot x}=e^{\imath \int_{-t}^{t} W_{\omega}(s, x) d s} .
$$

This lemma can be proved in the same way as in the proof of Theorem 3.1 in [J]. So, we only give a sketch of the proof.

Sketch of the proof. The Dyson expansion

$$
\begin{aligned}
& e^{-i E \omega \cdot x} e^{i t H_{0}} U_{A}(t,-t) e^{\imath t H_{0}} e^{i E \omega \cdot x} \\
& =I+\sum_{n=1}^{\infty}(-i)^{n} \int_{-t<t_{1}<\cdots<t_{n}<t} d t_{n} \cdots d t_{1} R_{E}\left(t_{n}\right) \cdots R_{E}\left(t_{1}\right)
\end{aligned}
$$

converges in the operator norm of $\mathscr{H}$ uniformly in large $E>0$, where

$$
R_{E}(t):=e^{-t E \omega \cdot x} e^{\imath t H_{0}} Q(t, \cdot) e^{-t t H_{0}} e^{\imath E \omega \cdot x} .
$$

Using

$$
s-\lim _{E \rightarrow+\infty}\left[e^{-\imath E \omega \cdot x} e^{-\imath s H_{0}} e^{\imath E \omega \cdot x}-e^{-\imath s c(E+\omega \cdot D) \alpha \cdot \omega}\right]=0,
$$

we can see that

$$
s-\lim _{E \rightarrow+\infty} \int_{-t}^{t} R_{E}(s) d s=-\int_{-t}^{t} W_{\omega}(s, \cdot) d s
$$

In the same way we also have

$$
s-\lim _{E \rightarrow+\infty} \int_{-t<t_{1}<\cdots<t_{n}<t} d t_{n} \cdots d t_{1} R_{E}\left(t_{n}\right) \cdots R_{E}\left(t_{1}\right)
$$




$$
\begin{aligned}
& =(-1)^{n} \int_{-t<t_{1}<\cdots<t_{n}<t} d t_{n} \cdots d t_{1} W_{\omega}\left(t_{n}, \cdot\right) \cdots W_{\omega}\left(t_{1}, \cdot\right) \\
& =\frac{(-1)^{n}}{n !}\left(\int_{-t}^{t} W_{\omega}(s, \cdot) d s\right)^{n},
\end{aligned}
$$

where we have used $\left[W_{\omega}(t, \cdot), W_{\omega}(s, \cdot)\right]=0$ for $s, t \in \mathbb{R}$. Thus (2.23) follows from (2.24) and (2.27).

In the momentum space it can be easily seen that

$$
s-\lim _{E \rightarrow+\infty} e^{-\imath E \omega \cdot x} P_{ \pm} e^{\imath E \omega \cdot x}=\frac{1}{2}(I \pm \alpha \cdot \omega)=P_{ \pm}(\omega) .
$$

From this together with Lemma 2.5 it follows that

$$
\begin{aligned}
& \lim _{E \rightarrow+\infty} e^{-\imath E \omega \cdot x} P_{ \pm} e^{\imath t H_{0}} U_{A}(t,-t) e^{\imath t H_{0}} P_{ \pm} e^{\imath E \omega \cdot x} \\
& =e^{\imath \int_{-1}^{t}\langle(\mp \omega), A(s, x \pm c s \omega)\rangle_{R} \cdot d s} P_{ \pm}(\omega) .
\end{aligned}
$$

Proof of Proposition 2.2. Let $f, g \in \mathscr{H}$ and define

$$
S_{\bar{E}}^{ \pm}(t):=e^{-\imath E \omega \cdot x} P_{ \pm} e^{\imath t H_{0}} U_{A}(t,-t) e^{\imath t H_{0}} P_{ \pm} e^{i E \omega \cdot x}
$$

and write

$$
\begin{aligned}
& \left(e^{-\imath E \omega \cdot x} P_{ \pm} S_{A}(0) P_{ \pm} e^{\imath E \omega \cdot x} f, g\right)-\left(e^{\imath K_{A}^{ \pm \omega}(0, x)} P_{ \pm}(\omega) f, g\right) \\
& \quad=\left(\left(e^{-\imath E \omega \cdot x} P_{ \pm} S_{A}(0) P_{ \pm} e^{\imath E \omega \cdot x}-S_{E}^{ \pm}(t)\right) f, g\right) \\
& \quad+\left(\left(S_{E}^{ \pm}(t)-e^{2 \int_{-t}^{\imath}\langle(\mp \omega), A(s, x \pm c s \omega)\rangle_{R^{\prime}} d s} P_{ \pm}(\omega)\right) f, g\right) \\
& \quad+\left(\left(e^{\imath \int_{-t}^{t}\langle(\mp \omega), A(s, x \pm c s \omega)\rangle_{R^{*}} d s}-e^{\imath K_{A}^{ \pm \omega}(0, x)}\right) P_{ \pm}(\omega) f, g\right) .
\end{aligned}
$$

The first term tends to zero as $t \rightarrow+\infty$ uniformly in large $E>0$ by Lemma 2.4 and the second term tends to zero as $E \rightarrow+\infty$ for each $t>0$ by (2.29). Since $\pm \omega \in S^{2} \backslash C_{A}$, the function $A(s, x \pm c s w)$ of $s$ is integrable for each $x \in \mathbb{R}^{3}$. Therefore, the third term goes to zero as $t \rightarrow+\infty$. Hence, we have proved Proposition 2.2 for $s=0$. Recalling (1.2) and noting that (see (2.25) and $(2.28))$

$$
s-\lim _{E \rightarrow+\infty}\left[e^{-\imath E \omega \cdot x} P_{ \pm} e^{\imath H_{0} e_{0}} e^{\imath \omega \omega x}-P_{ \pm}(\omega) e^{ \pm i s(c E+c \omega \cdot D)}\right]=0
$$

we have

$$
\begin{aligned}
w & -\lim _{E \rightarrow+\infty} e^{-\imath E \omega \cdot x} P_{ \pm} S_{A}(s) P_{ \pm} e^{\imath E \omega \cdot x} \\
& =P_{ \pm}(\omega) e^{\mp \imath s c \omega \cdot D} e^{\imath K_{A}^{ \pm \omega}(0, x)} e^{ \pm \imath s c \omega \cdot D} P_{ \pm}(\omega) \\
& =e^{\imath K_{A}^{ \pm \omega}(0, x \pm s c \omega)} P_{ \pm}(\omega) \\
& =e^{\imath K_{A}^{ \pm \omega}(s, x)} P_{ \pm}(\omega)
\end{aligned}
$$

This completes the proof. 
Here we give the idea of the proof of Theorem 1.3 for a simple case, $A=$ $(\phi, 0), \phi \in \mathcal{\&}$. In this case, the right hand side of (2.18) determines

$$
\mathbb{K}_{A}^{\omega}(\eta):=\int_{-\infty}^{\infty} \phi(t \widetilde{\omega}+\eta) d t
$$

since $K_{A}^{\omega}(\eta) \rightarrow 0$ as $|\eta| \rightarrow \infty$ with $\langle\eta, \widetilde{\omega}\rangle=0$. Thus the Fourier transform and the inverse Fourier transform yield

$$
\begin{aligned}
& \widehat{\phi}(\Xi)=(2 \pi)^{-2} \sqrt{1+c^{2}} \int_{\Pi_{\tilde{\omega}}} e^{-\imath\langle\eta, \Xi\rangle} K_{A}^{\omega}(\eta) d \eta, \text { for } \quad \Xi \in \Pi_{\widetilde{\omega}} \\
& K_{A}^{\omega}(\eta)=\left(2 \pi \sqrt{1+c^{2}}\right)^{-1} \int_{\Pi_{\tilde{\omega}}} e^{\imath\langle\eta, \Xi\rangle} \widehat{\phi}(\Xi) d \Xi, \quad \text { for } \quad \eta \in \mathbb{\Pi}_{\widetilde{\omega}}
\end{aligned}
$$

where $\mathbb{\Pi}_{\theta}:=\left\{\eta \in \mathbb{R}^{4} ;\langle\theta, \eta\rangle_{R^{4}}=0\right\}$. On the other hand, we can easily verify that

$$
\bigcup_{\omega \in S^{2}} \Pi_{\widetilde{\omega}}=\bar{D}, \text { the closure of } D .
$$

Therefore it follows that the only $\left.\widehat{\phi}\right|_{\bar{D}}$ is reconstructed from the right hand side of (2.18).

Remark. The potential $\phi$ can be completely reconstructed in the case of Schrödinger equations by a similar method [We]. Indeed, we obtain line integrals $\int_{-\infty}^{\infty} \phi\left(\eta_{0}, t \omega+\eta^{\prime}\right) d t, \eta=\left(\eta_{0}, \eta^{\prime}\right) \in \mathbb{R} \times \mathbb{R}^{3}$, instead of $K_{A}^{\omega}(\eta)$, from the scattering operator in the case of Schrödinger equations. Then, using the same argument as above, we see that these line integrals determine the whole $\widehat{\phi}$. Hence the potential $\phi$ is completely reconstructed.

Now we define

$$
\widetilde{C_{A}}:=C_{A} \cup\left(\bigcup_{k=1}^{N^{\prime}} C\left(\mathscr{L}_{k}^{\prime}\right)\right)
$$

Lemma 2.6. Let $\omega \in S^{2} \backslash \widetilde{C_{A}}$ and $\theta \in S^{3}$ such that $\widetilde{\omega}$ and $\theta$ are linearly independent, and let $\eta \in \mathbb{R}^{4}$. Then one has

$$
\left.\frac{d}{d s} K_{A}^{\omega}(s \theta+\eta)\right|_{s=0}=-\int_{-\infty}^{+\infty}\left\langle\widetilde{\omega} \wedge \theta, F_{A}(t \widetilde{\omega}+\eta)\right\rangle_{R^{t}} d t
$$

where $X \wedge Y=\left(X_{j} Y_{k}-X_{k} Y_{\jmath}\right)_{0 \leq \jmath<{ }_{k} \leq 3} \in \mathbb{R}^{6}$ for $X=\left(X_{0}, \cdots, X_{3}\right)$ and $Y=\left(Y_{0}, \cdots, Y_{3}\right)$.

Remark. Since $\widetilde{\omega} \in S^{2} \backslash \widetilde{C_{A}}, F_{A}(t \widetilde{\omega}+\eta)$ is integrable with respect to $t \in \mathbb{R}$.

Proof. By Stokes' theorem we have

$$
K_{A}^{\omega}(\eta)-K_{A}^{\omega}\left(\eta+s_{0} \theta\right)=\int_{0}^{s_{0}} d s \int_{-\infty}^{\infty}\left\langle\widetilde{\omega} \wedge \theta, F_{A}(t \widetilde{\omega}+s \theta+\eta)\right\rangle_{R^{\sigma}} d t,
$$


from which the lemma follows immediately.

Since

$$
\frac{d}{d s} \exp \left(-i K_{A}^{\omega}(s \theta+\eta)\right)=-i \frac{d}{d s} K_{A}^{\omega}(s \theta+\eta) \cdot \exp \left(-i K_{A}^{\omega}(s \theta+\eta)\right),
$$

we can conclude from Proposition 2.2 and Lemma 2.6 that for each $\eta \in \mathbb{R}^{4}, \omega \in$ $S^{2} \backslash \widetilde{C_{A}}$ and $\theta \in S^{3}$ the integral

$$
\int_{-\infty}^{+\infty}\left\langle\widetilde{\omega} \wedge \theta, F_{A}(t \widetilde{\omega}+\eta)\right\rangle_{R^{6}} d t
$$

can be constructed from the scattering operator $S_{A}(0)$ (see (1.2)).

Proof of Theorem 1.3. For a while we fix $\Xi_{0}=\left(\tau_{0}, \xi_{0}\right) \in D \backslash \Sigma$. Since $C\left(\Xi_{0}\right) \cap$ $\widetilde{C_{A}}$ is a finite or empty set due to $(2.16)$, we can take $\left\{\omega_{j}^{0}\right\}_{j=1}^{3} \subset C\left(\Xi_{0}\right) \backslash \widetilde{C}_{A}$ with $\omega_{j}^{0} \neq \omega_{k}^{0}$ if $j \neq k$. Then $\left\{\widetilde{\omega}_{j}^{0}\right\}_{j=1}^{3}$ are linearly independent in $\mathbb{R}^{4}$, where $\widetilde{\omega}_{j}^{0}=$ $\left(1, c \omega_{j}^{0}\right)$. We also take $\widetilde{\omega}_{0}^{0}$ from $\boldsymbol{R}^{4}$ so that $\left\{\widetilde{\omega}_{j}^{0}\right\}_{j=0}^{3}$ is a basis of $\mathbb{R}^{4}$. (Here we abused the notation $\widetilde{\omega}_{0}^{0}$, which need not be expressed as $\left(1, c \omega_{0}^{0}\right)$ for some $\omega_{0}^{0} \in$ $S^{2}$, to simplify notations below.) It should be notice that $\left\{\widetilde{\omega}_{j}^{0} \wedge \widetilde{\omega}_{k}^{0}\right\}_{0 \leq k<j \leq 3}$ is also a basis of $\boldsymbol{R}^{6}$.

We first assume $F_{A} \in L^{1}\left(\mathbb{R}_{t} \times \mathbb{R}_{x}^{3}\right)$. Noting that $\left\langle\Xi_{0}, \widetilde{\omega}_{j}^{0}\right\rangle_{R^{4}}=0$ for $j=1,2,3$, we have, for each $0 \leq k<j \leq 3$,

$$
\begin{aligned}
& \left\langle\widetilde{\omega}_{j}^{0} \wedge \widetilde{\omega}_{k}^{0}, \widehat{F_{A}}\left(\Xi_{0}\right)\right\rangle_{R^{6}} \\
& \quad=(2 \pi)^{-2} \sqrt{1+c^{2}} \int_{\Pi \widetilde{\omega}_{1}^{0}} e^{-\imath\left\langle\eta, \Xi_{0}\right\rangle} d \eta \int_{-\infty}^{\infty}\left\langle\widetilde{\omega}_{j}^{0} \wedge \widetilde{\omega}_{k}^{0}, F_{A}\left(t \widetilde{\omega}_{j}^{0}+\eta\right)\right\rangle_{R^{6}} d t .
\end{aligned}
$$

Since $\left\{\widetilde{\omega}_{j}^{0} \wedge \widetilde{\omega}_{k}^{0}\right\}_{0 \leq k<j \leq 3}$ is a basis of $\mathbb{R}^{6}$ and since the integral with respect to $t$ in the right hand side is determined by the scattering operator, $\widehat{F_{A}}\left(\Xi_{0}\right)$ is determined by the scattering operator for each $\Xi_{0} \in D \backslash \Sigma$.

If $F_{A}$ does not belong to $L^{1}\left(\mathbb{R}_{t} \times \mathbb{R}_{x}^{3}\right)$, we can not use the above formula directly since $\widehat{F_{A}}$ should be regarded as a distribution. So, more complicated arguments are needed to represent $\widehat{F_{A}}$ in terms of the line integrals $(2.31)$ as in [St].

We set $c=1$ for simplicity, and fix $j=1,2,3$ for a while. Since $\omega_{j}^{0}$ and $\xi_{0}$ are not parallel, they span a two-dimensional subspace, denoted by $\Lambda_{j}$, in $\mathbb{R}_{\xi}^{3}$. We denote by $\Pi_{j}$ the orthogonal complement of $\Lambda_{j}$ in $\boldsymbol{R}_{\xi}^{3}, \mathbb{R}_{\xi}^{3}=\Lambda, \oplus \Pi_{j}$, and denote by $\xi^{\prime}, \xi^{\prime \prime}$ the $\Lambda_{j}, \Pi_{j}$-component of $\xi \in \mathbb{R}_{\xi}^{3}$, respectively.

We can find a unique $\chi_{j}^{0} \in \Lambda_{j}$, such that $\left\{\omega_{j}^{0}, \chi_{j}^{0}\right\}$ is an orthonormal basis of 
$\Lambda_{j}$ with $\left.\left\langle\chi_{j}^{0}, \xi_{0}\right\rangle_{R^{3}}\right\rangle 0$. Then we can write $\xi_{0}=-\tau_{0} \omega_{j}^{0}+\lambda_{j}^{0} \chi_{j}^{0}$, where $\lambda_{j}^{0}:=\mid \xi_{0}+$ $\tau_{0} \omega_{j}^{0} \mid>0$.

Now we define a family of orthonormal bases $\left\{\omega_{j}(\theta), \chi_{j}(\theta)\right\},-\pi / 4<\theta$ $<\pi / 4$, of $\Lambda_{j}$ by rotating the basis $\left\{\omega_{j}^{0}, \chi_{j}^{0}\right\}$ :

$$
\begin{aligned}
& \omega_{j}(\theta):=\omega_{j}^{0} \cos \theta+\chi_{j}^{0} \sin \theta, \\
& \chi_{j}(\theta):=-\omega_{j}^{0} \sin \theta+\chi_{j}^{0} \cos \theta .
\end{aligned}
$$

It is easily seen that if $(\tau, \xi)$ is sufficiently near $\left(\tau_{0}, \xi_{0}\right)$ there exists a unique $\theta_{j}(\tau, \xi) \in \mathbb{R}$ near 0 satisfying $\left\langle\xi^{\prime}, \omega_{j}\left(\theta_{j}(\tau, \xi)\right)\right\rangle_{R^{s}}=-\tau$. We set $\lambda_{j}(\tau, \xi)$ $:=\left\langle\xi^{\prime}, \chi_{j}\left(\theta_{j}(\tau, \xi)\right)\right\rangle_{R^{3}}=\sqrt{\left|\xi^{\prime}\right|^{2}-\tau^{2}}$ and $\rho_{j}(\tau, \xi):=\left\langle\xi^{\prime \prime}, \varpi_{j}^{0}\right\rangle$, where $\varpi_{j}^{0}$ is a unit vector in $\Pi_{\text {, }}$.

Consequently we can see that there are a small neighborhood $U_{0}$ of $\left(\tau_{0}, \xi_{0}\right) \in \mathbb{R} \times \mathbb{R}^{3}$, a small neighborhood $V_{j} \subset\left\{\left(\theta_{j}, \lambda_{j}, \rho_{j}, \tau_{j}\right) \in(-\pi / 4, \pi / 4) \times \mathbb{R} \times \mathbb{R}\right.$ $\times \mathbb{R}\}$ of $\left(0, \lambda_{j}^{0}, 0, \tau_{0}\right)$ and a diffeomorphism

$$
\left(\theta_{j}, \lambda_{j}, \rho_{j}, \tau_{j}\right): U_{0} \longrightarrow V
$$

such that

$$
\begin{aligned}
& \theta_{j}\left(\tau_{0}, \xi_{0}\right)=0, \quad \lambda_{j}\left(\tau_{0}, \xi_{0}\right)=\lambda_{j}^{0}, \quad \rho_{j}\left(\tau_{0}, \xi_{0}\right)=0, \quad \tau_{j}\left(\tau_{0}, \xi_{0}\right)=\tau_{0}, \\
& \tau_{j}(\tau, \xi)=\tau, \quad-\tau_{j}(\tau, \xi) \omega_{j}\left(\theta_{j}(\tau, \xi)\right)+\lambda_{j}(\tau, \xi) \chi_{j}(\theta,(\tau, \xi))+\rho,(\tau, \xi) \varpi_{j}^{0}=\xi
\end{aligned}
$$

We also note that $\left|\partial(\tau, \xi) / \partial\left(\theta_{j}, \lambda_{j}, \rho_{j}, \tau_{j}\right)\right|=\lambda_{j}$.

Since $\left\{\widetilde{\omega}_{k}^{0} \wedge \widetilde{\omega}_{j}^{0}\right\}_{0 \leq k<j \leq 3}$ is a basis of $\mathbb{R}^{6}$ and since each function $\omega_{j}\left(\theta_{j}(\tau, \xi)\right)$ is smooth on $U_{0}$ with $\omega_{j}\left(\theta_{j}\left(\tau_{0}, \xi_{0}\right)\right)=\omega_{j}^{0},\left\{\widetilde{\omega}_{k}^{0} \wedge \widetilde{\omega}_{,}\left(\theta_{j}(\tau, \xi)\right)\right\}_{0 \leq k<j \leq 3}$ is also a basis of $\mathbb{R}^{6}$ for each $(\tau, \xi)$ in a small neighborhood of $\left(\tau_{0}, \xi_{0}\right)$, which can be assumed to be $U_{0}$, where $\widetilde{\omega}_{j}(\theta):=(1, \omega,(\theta))$. We can also assume that $\omega_{,}\left(\theta_{j}(\tau, \xi)\right) \notin \widetilde{C_{A}}$ for $(\tau, \xi) \in U_{0}, 1 \leq j \leq 3$. Thus, for any $\phi \in C_{0}^{\infty}\left(U_{0} ; \mathbb{C}^{6}\right)$ there exist $\phi_{j k}(\tau, \xi) \in C_{0}^{\infty}\left(U_{0} ; \mathbb{C}\right), 0 \leq k<j \leq 3$, such that

$$
\phi(\tau, \xi)=\sum_{0 \leq k<j \leq 3} \phi_{j k}(\tau, \xi) \widetilde{\omega}_{k}^{0} \wedge \widetilde{\omega}_{j}\left(\theta_{j}(\tau, \xi)\right)
$$

on $U_{0}$. So we have

$$
\begin{aligned}
& \left\langle\widehat{F_{A}}, \phi\right\rangle=\left\langle F_{A}, \widehat{\phi}\right\rangle \\
& =\iint d t d x\left\langle F_{A}(t, x),(2 \pi)^{-2} \iint e^{-\imath t \tau-\imath\langle x, \xi)} \phi(\tau, \xi) d \tau d \xi\right\rangle_{C^{6}} \\
& =(2 \pi)^{-2} \sum_{0 \leq k<j \leq 3} \iint d t d x\left\langle F_{A}(t, x), \iint e^{-\imath t \tau-\imath\langle x, \xi\rangle} \phi_{j k}(\tau, \xi) \widetilde{\omega}_{k}^{0} \wedge \widetilde{\omega}_{j}\left(\theta_{j}(\tau, \xi)\right) d \tau d \xi\right\rangle_{C^{6}} \\
& =(2 \pi)^{-2} \sum_{0 \leq k<j \leq 3} \iint d t d x\left\langle F_{A}(t, x),\right.
\end{aligned}
$$




$$
\begin{aligned}
& \left.\iiint \int e^{-t t \tau_{j}-\imath\left\langle x, \xi\left(\theta_{j,}, \lambda_{j}, \rho_{,}, \tau_{j}\right)\right\rangle} \phi_{j k}\left(\tau_{j}, \xi\left(\theta_{j}, \Lambda_{j}, \rho_{j}, \tau_{j}\right)\right) \widetilde{\omega}_{k}^{0} \wedge \widetilde{\omega}_{j}\left(\theta_{j}\right) \lambda_{j} d \tau_{j} d \rho_{j} d \lambda_{j} d \theta_{j}\right\rangle_{C^{\circ}} \\
= & (2 \pi)^{-2} \sum_{0 \leq k<j \leq 3} \iint d t d x \int G_{j k}(t, x, \theta) d \theta_{j},
\end{aligned}
$$

where

$$
\begin{aligned}
G_{j k}^{0}\left(t, x, \theta_{j}\right) & :=\left\langle F_{A}(t, x), \widetilde{\omega}_{k}^{0} \wedge \widetilde{\omega}_{j}\left(\theta_{j}\right)\right\rangle_{C^{6},} \\
G_{j k}^{1}\left(t, x, \theta_{j}\right) & :=\iiint e^{-\imath t \tau_{j}-\imath\left\langle x, \xi\left(\theta_{j}, \lambda_{,}, \rho_{j}, \tau_{j}\right)\right\rangle} \phi_{j k}\left(\tau_{j}, \xi\left(\theta_{j}, \lambda_{j}, \rho_{j}, \tau_{j}\right)\right) \lambda_{j} d \tau_{j} d \rho_{j} d \lambda_{j}, \\
G_{j k}\left(t, x, \theta_{j}\right) & =G_{j k}^{0}\left(t, x, \theta_{j}\right) G_{j k}^{1}\left(t, x, \theta_{j}\right) .
\end{aligned}
$$

Integrating by parts with respect to $\left(\tau_{j}, \rho_{j}, \lambda_{j}\right)$ in $(2.32)$, we can see that for any $n>0$ there exists $K_{n}$ such that

$$
\left|G_{j k}\left(t, x, \theta_{j}\right)\right| \leq K_{n}\left(1+\left|t-\left\langle\omega_{j}\left(\theta_{j}\right), x\right\rangle\right|+\left|\left\langle\chi_{j}\left(\theta_{j}\right), x\right\rangle\right|+\left|\left\langle\omega_{j}^{0}, x\right\rangle\right|\right)^{-n}\left|F_{A}(t, x)\right|
$$

uniformly in $\left(t, x, \theta_{j}\right)$. We will show that.

$$
\sup _{\theta,}\left\|G_{, k}\left(\cdot, \cdot, \theta_{j}\right)\right\|_{L^{1}\left(R_{t} \times R_{x}^{3}\right)}<+\infty .
$$

Let $X\left(\theta_{\jmath}\right)$ be the orthogonal projection of $X=(t, x)$ onto $\prod_{\widetilde{\omega},\left(\theta_{j}\right)}$. Then, observing that

$$
\left\{\left(1,-\omega_{j}\left(\theta_{j}\right)\right),\left(0, \chi_{j}\left(\theta_{j}\right)\right),\left(0, \varpi_{j}^{0}\right)\right\}
$$

is a basis of $\Pi_{\widetilde{\omega},\left(\theta_{1}\right)}$, we have

$$
\begin{aligned}
\left|G_{j k}\left(t, x, \theta_{\jmath}\right)\right| & \leq K_{n}^{\prime}\left(1+\left|X\left(\theta_{\jmath}\right)\right|\right)^{-n}\left|F_{A}(X)\right| \\
& \leq K_{n}^{\prime}\left(1+\left|X\left(\theta_{\jmath}\right)\right|\right)^{-n} \sum_{k=1}^{N^{\prime}} g_{F_{k}^{\prime}}^{L_{k}^{\prime}}\left(\left|X_{L_{k}^{\prime}}\right|\right)
\end{aligned}
$$

uniformly in $\left(t, x, \theta_{j}\right)$ for some $K_{n}^{\prime}>0$. Here note that $L_{k}^{\prime} \varangle \Pi_{\widetilde{\omega},\left(\theta_{j}\right)}$ for all $k$ since $\omega,\left(\theta_{j}(\tau, \xi)\right) \notin \widetilde{C_{A}}$ if $(\tau, \xi) \in U_{0}, 1 \leq j \leq 3$. Thus, (2.34) implies (2.33). Thus, Fubini's theorem guarantees that

$$
\iint d t d x \int G_{\jmath k}\left(t, x, \theta_{\jmath}\right) d \theta_{j}=\int d \theta_{\jmath} \iint G_{j k}\left(t, x, \theta_{\jmath}\right) d t d x
$$

Now for each $\theta$, we introduce new coordinates $\left\{s_{j}, \lambda_{j}^{\prime}, \rho_{j}^{\prime}, \tau_{j}^{\prime}\right\}$ for $\{t, x\}$ :

$$
t=\tau_{j}^{\prime}+s_{j}, \quad x=\tau_{j}^{\prime} \omega_{j}\left(\theta_{j}\right)+\lambda_{j}^{\prime} \chi_{\jmath}\left(\theta_{j}\right)+\rho_{j}^{\prime} \varpi_{j}^{0}
$$

where $s_{j}, \tau_{j}^{\prime}, \lambda_{\jmath}^{\prime}, \rho_{j}^{\prime} \in \boldsymbol{R}$. Then

$$
e^{-\imath t \tau-l x \cdot \xi\left(\theta_{1}, \lambda_{1}, \rho_{1}, \tau_{1}\right)}=e^{-\imath\left(s, \tau+\lambda_{3}^{\prime} \lambda_{1}+\rho_{,}, \rho_{1}\right)}
$$

is independent of $\tau_{j}^{\prime}$, and so is $G_{j k}^{1}$. Thus, the right hand side of (2.35) is written as 


$$
\int d \theta_{j} \iiint G_{j k}^{1} d s_{j} d \lambda_{j}^{\prime} d \rho_{j}^{\prime} \int_{-\infty}^{\infty}\left\langle F_{A}\left(\tau_{j}^{\prime}+s_{j}, \tau_{j}^{\prime} \omega_{j}\left(\theta_{j}\right)+\lambda_{j}^{\prime} \chi_{j}\left(\theta_{j}\right)+\rho_{j}^{\prime} \varpi_{j}^{0}\right), \widetilde{\omega}_{k}^{0} \wedge{\widetilde{\omega_{j}}}_{j}\left(\theta_{j}\right)\right\rangle_{C^{6}} d \tau_{j}^{\prime}
$$

Therefore we have

$$
\begin{aligned}
& \left\langle\widehat{F_{A}}, \phi\right\rangle \\
& =(2 \pi)^{-2} \sum_{0 \leq k<j \leq 3} \int d \theta_{j} \iiint G_{j k}^{1} d s_{j} d \lambda_{j}^{\prime} d \rho_{j}^{\prime} \\
& \quad \times \int_{-\infty}^{\infty}\left\langle\mathbb{F}_{A}\left(\tau_{j}^{\prime} \widetilde{\omega}_{j}\left(\theta_{j}\right)+\eta\left(\lambda_{j}^{\prime}, s_{j}, \rho_{j}^{\prime}, \theta_{j}\right)\right), \widetilde{\omega}_{k}^{0} \wedge \widetilde{\omega}_{j}\left(\theta_{j}\right)\right\rangle_{C^{6}} d \tau_{j}^{\prime},
\end{aligned}
$$

where $\eta\left(\lambda_{j}^{\prime}, s_{j}, \rho_{j}^{\prime}, \theta_{j}\right)=\left(s_{j}, \lambda_{j}^{\prime} \chi,\left(\theta_{j}\right)+\rho_{j}^{\prime} \bar{\omega}_{j}^{0}\right)$. Since $\left(\tau_{0}, \xi_{0}\right) \in D \backslash \Sigma$ is arbitrary and $G_{j k}^{1}$ is independent of $F_{A}$, we have completed the proof of Theorem 1.3.

\section{§. Proofs of Theorems $1.5,1.7$ and Corollary 1.6}

Lemma 3.1. Suppose that $F$ is a bounded function on $\mathbb{R}^{d}, d \geq 2$, such that

$$
|F(x)| \leq g\left(\left|\langle V, x\rangle_{R^{d}}\right|\right) \text { on } \mathbb{R}^{d},
$$

for some $V \in S^{d-1}$ and $g$ with $g(t)(1+t)^{-1} \in L^{1}((0, \infty))$. Let $\chi_{\varepsilon}(\xi):=\chi(\xi / \varepsilon), \varepsilon>0$, for $\chi \in \&\left(\mathbb{R}^{d}\right)$ and let $\widehat{F}$ be the Fourier transform of $\mathbb{F}$. Then

$$
\lim _{\varepsilon \downarrow 0}\left\langle\widehat{F}, \chi_{\varepsilon} u\right\rangle=0
$$

for any $u \in \mathbb{C}_{0}^{\infty}\left(\mathbb{R}^{d}\right)$.

Proof. We assume $V=(1,0,0,0)$ for simplicity and calculate

$$
\begin{aligned}
\left\langle\widehat{F}, \chi_{\varepsilon} u\right\rangle & =\left\langle F, \widehat{\chi_{\varepsilon} u}\right\rangle \\
& =\text { const. }\langle F, \widehat{\chi} \varepsilon * \widehat{u}\rangle \\
& =\text { const. } \iint \varepsilon^{d} F(x) \widehat{\chi}(\varepsilon(x-y)) \widehat{u}(y) d y d x .
\end{aligned}
$$

Using the estimate $|\widehat{\chi}(x)| \leq K(\mathbb{1}+|x|)^{-d}$, we get $\left|\varepsilon^{d} \widehat{\chi}(\varepsilon x)\right| \leq K(1+|x|)^{-d}$ uniformly in $\varepsilon \in(0,1)$. Therefore it follows that

$$
\begin{aligned}
& \left|\varepsilon^{d} F(x) \widehat{\chi}(\varepsilon(x-y)) \widehat{u}(y)\right| \\
\leq & K|F(x)|(1+|x-y|)^{-d}|\widehat{u}(y)| \\
\leq & K g\left(\left|x_{1}\right|\right)(1+|x|)^{-d}(1+|y|)^{d}|\widehat{u}(y)|,
\end{aligned}
$$

where we have used (3.1) in the last step. On the other hand, denoting $x=$ $\left(x_{1}, x^{\prime}\right)$, we see that

$$
\int\left(1+\left|x_{1}\right|+\left|x^{\prime}\right|\right)^{-d} d x^{\prime} \leq \text { const. }\left(1+\left|x_{1}\right|\right)^{-1}
$$


Thus, $g\left(\left|x_{1}\right|\right)(1+|x|)^{-d}(1+|y|)^{d}|\widehat{u}(y)| \in L^{1}\left(\boldsymbol{R}_{x}^{d} \times \boldsymbol{R}_{y}^{d}\right)$. By applying Lebesgue's convergence theorem to $(3.3),(3.2)$ follows.

Proof of Theorem 1.5. Let $\phi \in C_{0}^{\infty}\left(\boldsymbol{R}^{4} \backslash\{0\} ; \boldsymbol{C}^{6}\right)$. Due to $(1.5)$, we can take $\chi \in C^{\infty}\left(\mathbb{R}^{4}\right)$ such that

$$
\operatorname{supp} \chi \phi \subset D, \operatorname{supp}\{(1-\chi) \phi\} \cap \operatorname{supp} \widehat{F_{A}}=\emptyset .
$$

Therefore,

$$
\left\langle\widehat{F_{A}}, \phi\right\rangle=\left\langle\widehat{F_{A}}, \chi \phi\right\rangle \text {. }
$$

Thus Theorem 1.3 guarantees that $\left.\widehat{F_{A}}\right|_{R^{4} \backslash\{0\}}$ can be reconstructed from the scattering operator. Hence, by Lemma 3.1 we see that $\widehat{F_{A}}$ and so $F_{A}$ can be reconstructed from scattering operator, since each $F_{k}$ satisfies (3.1).

Proof of Corollary 1.6. If $F_{k}$ satisfies (1.6), the support of $\widehat{F_{K}}$ is contained in the three-dimensional subspace

$$
V_{k}^{\perp}:=\left\{\Xi \in \mathbb{R}^{4} ;\left\langle V_{k}, \Xi\right\rangle=0\right\},
$$

which satisfies $V_{k}^{\perp} \backslash\{0\} \subset D$ if $V_{k} \in T$.

Proof of Theorem 1.7. (1) Let $L$ be the one-dimensional subspace spanned by $V$ and fix $\Xi_{0} \notin L$. Then it is easy to see that there exist a neighborhood $U_{0}$ of $\Xi_{0}, \sigma_{0} \subset \mathbb{R}$ and $\varepsilon_{0}>0$ such that

$$
U_{0} \cap L=\emptyset, \quad U_{0}+\sigma V \subset D \backslash \Sigma \text { if } \quad\left|\sigma-\sigma_{0}\right|<\varepsilon_{0} .
$$

We define $\phi_{\sigma}(\Xi):=\phi(\Xi-\sigma V)$ for any $\phi \in C_{0}^{\infty}\left(U_{0}\right)$ and $\sigma \in \mathbb{R}$. Then $\phi_{\sigma} \in C_{0}^{\infty}\left(U_{0}\right.$ $+\sigma V) \in C_{0}^{\infty}(D \backslash \Sigma)$ if $\left|\sigma-\sigma_{0}\right|<\varepsilon_{0}$. Therefore Theorem 1.3 says that $\left\langle\widehat{F_{A}}, \phi_{\sigma}\right\rangle$ can be reconstructed from $S_{A}(0)$. On the other hand,

$$
f(\sigma):=\left\langle\widehat{F_{A}}, \phi_{\sigma}\right\rangle=\left\langle F_{A} e^{-\imath\langle X, \sigma V\rangle}, \widehat{\phi}\right\rangle
$$

is an analytic function on $\{\sigma \in \mathbb{C} ; \operatorname{Re} \sigma \in \mathbb{R},|\operatorname{Im} \sigma|<\delta\}$ by (1.8). Hence, $f(\sigma)$ is determined by $S_{A}(0)$ for any $\sigma \in \mathbb{R}$, and so is $f(0)=\left\langle\widehat{F_{A}}, \phi\right\rangle$, in particular. Since $\Xi_{0}$ is arbitrary, this implies that $\left.\widehat{F_{A}}\right|_{R^{4} \backslash L}$ is determined by $S_{A}(0)$.

(2) Let $L$ be the one-dimensional subspace spanned by $V$ and take $\phi \in$ $C^{\infty}\left(L^{\perp}\right)$ with $\phi(\eta)=1$ for $|\eta|>2$ and $\phi(\eta)=0$ for $|\eta|<1$, and define $\phi_{\varepsilon}(\eta)=$ $\phi(\eta / \varepsilon)$ for $\varepsilon>0$. It suffices to show that

$$
\lim _{\varepsilon \downarrow 0} \phi_{\varepsilon}(\eta) \widehat{F_{A}^{j k}}=\widehat{F_{A}^{j k}} \text { in } \mathscr{D}^{\prime}\left(\boldsymbol{R}^{4}\right), \quad 0 \leq j<k \leq 3,
$$


since $\operatorname{supp}\left(\phi_{\varepsilon} u\right) \subset \mathbb{R}^{4} \backslash \mathbb{L}$ for all $u \in C_{0}^{\infty}$. To do so we assume $V=(1,0,0,0)$ for simplicity and write $X=(t, x)$. In the same way as in the proof of Lemma 3.1 it suffices to show that

$$
\begin{aligned}
& \iint e^{-\delta|t|} g\left(\left|\left\langle V^{\prime}, X\right\rangle\right|\right)(1+|x|)^{-3} d t d x \\
& \quad \leq \text { const. } \iint e^{-\delta|t| / 2} g\left(\left|\left\langle V^{\prime}, X\right\rangle\right|\right)(1+|X|)^{-3} d t d x
\end{aligned}
$$

is finite. But. this follows immediately.

\section{§4. Proof of Proposition 1.4}

Proof of Proposition 1.4. Since $\phi \in \mathscr{S}, S_{\lambda A}(0)$ is real-analytic in $\lambda$ (cf.[Th], p.249]):

$$
S_{\lambda A}(0)=\sum_{n=0}^{\infty}(-\lambda)^{n} S_{n}
$$

where each $S_{n}$ is a bounded operator on $\mathscr{H}$ and

$$
S_{0}=I, \quad S_{1}=\int_{-\infty}^{\infty} e^{\imath t H_{0}} \phi(t, \cdot) e^{-\imath t H_{0}} d t \cdot \cdots
$$

So, it suffices to show that $S_{1} \neq 0$.

We recall that the $4 \times 4$ matrix $h_{0}(\xi)=\alpha \cdot \xi+\beta$, the symbol of $H_{0}$, has the eigenvalues $\pm \sqrt{|\xi|^{2}+1}$ with multiplicity two, respectively, and the associated eigenprojections are denoted by $P^{ \pm}(\xi)$, and that $\mathbb{P}_{ \pm}$denotes the orthogonal projection in $\mathscr{H}$ onto the positive/negative energy subspace of $H_{0}: \mathbb{P}_{ \pm}=\frac{1}{2}(I \pm$ $\frac{H_{0}}{\left|H_{0}\right|} \mid$.

Then we have

$$
\mathscr{F}_{x \rightarrow \xi} P_{-} S_{1} P_{+} \mathscr{F}_{x \rightarrow \xi}^{-1}=\int_{-\infty}^{\infty} P^{-}(\xi) e^{-t t \sqrt{|\xi|^{2}+1}} \widetilde{\phi}(t) e^{-\imath t \sqrt{|\xi|^{2}+1}} \mathbb{P}^{+}(\xi) d t,
$$

where $\widetilde{\phi}(t)=\mathscr{F}_{x \rightarrow \xi} \phi(t, \cdot) \mathscr{F}_{x \rightarrow \xi}^{-1}$. Therefore $\mathscr{F}_{x \rightarrow \xi} P_{-} S_{1} P_{+} \mathscr{F}_{x \rightarrow \xi}^{-1}$ is the integral operator with kernel

$$
\begin{aligned}
T(p, q): & =(2 \pi)^{-3 / 2} \int_{-\infty}^{\infty} e^{-\imath t \sqrt{|p|^{2}+1}}\left(\mathscr{F}_{x \rightarrow \xi} \phi\right)(t, p-q) e^{-\imath t \sqrt{|q|^{2}+1}} P^{-}(p) P^{+}(q) d t \\
& =(2 \pi)^{-1} \widehat{\phi}\left(\sqrt{|p|^{2}+1}+\sqrt{|q|^{2}+1}, p-q\right) P^{-}(p) P^{+}(q) .
\end{aligned}
$$

Thus the proposition follows from Lemma 4.1 below since $\widehat{\phi}(\tau, \xi) \neq 0$ for some $(\tau, \xi) \in D_{1}$. 
Lemma 4.1. For each $(\tau, \xi) \in D_{1}, \xi \neq 0$, there exist $p$ and $q$ in $\boldsymbol{R}^{3}$ such that (i) $\xi=p-q$, (ii) $\tau=\sqrt{|p|^{2}+1}+\sqrt{|q|^{2}+1}$, (iii) $P^{-}(p) P^{+}(q) \neq 0$.

Proof of Lemma 3.1. We can write $2 \sqrt{1+r^{2}}=\tau$ for some constant $r>0$ because of $\tau>\sqrt{|\xi|^{2}+4}$. Since $|\xi| / r<2$, we can take $\omega, \theta \in S^{2}$ with $\omega-\theta=\xi / r$, and we define $p=r \omega, q=r \theta$. Then it is clear that they satisfy (i) and (ii). We next show that they satisfy (iii). Suppose that $P^{-}(p) P^{+}(q)=0$. Then $P^{+}(p) \supset$ $P^{+}(q)$. On the other hand, both operators have the same rank, $\operatorname{rank} P^{+}(p)=$ $\operatorname{rank} P^{+}(q)=2$, and so they coincide: $P^{+}(p)=P^{+}(q)$. Hence noting that $P^{+}(u)=$ $(1 / 2)\left(I+h_{0}(u) /\left|h_{0}(u)\right|\right)$ for each $u \in \mathbb{R}^{3}$ and that $\left|h_{0}(p)\right|=\left|h_{0}(q)\right|=\sqrt{1+r^{2}}$, we obtain $\alpha \cdot(p-q)=0$, and it follows that $p=q$. This contradicts with $\omega-\theta=\xi / r$ $\neq 0$. We have thus proved the lemma.

\section{§5. Relativistic Invariance}

A Lorentz transformation $\Lambda: \mathbb{R}^{4} \longrightarrow \mathbb{R}^{4}$ is a linear map preserving the Lorentz metric,

$$
\left\langle X, X^{\prime}\right\rangle_{L M}:=c^{2} x_{0} x_{0}^{\prime}-\sum_{j=1}^{3} x_{\rho} x_{\jmath}^{\prime}
$$

where $X=\left(x_{0}, \cdots, x_{3}\right)$, etc. This condition is equivalent to

$$
{ }^{t} \Lambda G \Lambda=G, \text { where } G:=\left(\begin{array}{cc}
c^{2} I_{1} & O \\
O & -I_{3}
\end{array}\right) .
$$

A Poincaré transformation,

$$
\mathbb{R}^{4} \ni X \mapsto X^{\prime}=\Lambda X+a,
$$

is the combination of a Lorentz transformation $\Lambda$ and a space-time translation by $a \in \mathbb{R}^{4}$ and is associated with changing an inertial frame with coordinate $X=$ ${ }^{t}(t, x)$ into another inertial frame with coordinate $X^{\prime}={ }^{t}\left(t^{\prime}, x^{\prime}\right)$ defined by (5.2). By this change the Dirac equation

$$
i \frac{\partial}{\partial t} \Psi(t, x)=\left[c \sum_{j=1}^{3} \alpha,\left(\frac{1}{i} \frac{\partial}{\partial x_{j}}-A^{j}(t, x)\right)+\alpha_{4} m c^{2}-A^{0}(t, x) I_{4}\right] \Psi(t, x)
$$

in the old inertial frame is converted into the Dirac equation

$$
i \frac{\partial}{\partial t^{\prime}} \Psi^{\prime}\left(t^{\prime}, x^{\prime}\right)=\left[c \sum_{j=1}^{3} \alpha_{j}\left(\frac{1}{i} \frac{\partial}{\partial x_{j}^{\prime}}-A^{* j}\left(t^{\prime}, x^{\prime}\right)\right)+\alpha_{4} m c^{2}-A^{* 0}\left(t^{\prime}, x^{\prime}\right) I_{4}\right] \Psi^{\prime}\left(t^{\prime}, x^{\prime}\right)
$$


in the new inertial frame. Each component of the potential $A^{*}\left(X^{\prime}\right)$ in the new frame is written as a linear combination of those of $A\left(\Lambda^{-1}\left(X^{\prime}-a\right)\right.$ ) (see, e.g., [Tha]). Let $V_{1}, \cdots, V_{n}$ be a basis of a subspace $L$. Then there exists a constant $K>0$ such that

$$
K^{-1}\left|X_{L}\right| \leq \sum_{j=1}^{n}\left|\left\langle V_{j}, X\right\rangle\right| \leq K\left|X_{L}\right|
$$

Hence we can see that $A^{*} \in S\left({ }^{t} \Lambda^{-1} L\right)$ if $A \in S(L)$. Each component $F_{A^{*}}^{* \operatorname{lm}}\left(X^{\prime}\right)$ of the electromagnetic field $F_{A^{*}}^{*}\left(X^{\prime}\right)$ in the new frame is also written as a linear combination of components $F_{A}^{j k}\left(\Lambda^{-1}\left(X^{\prime}-a\right)\right), 0 \leq j<k \leq 3$, of $F_{A}\left(\Lambda^{-1}\left(X^{\prime}-a\right)\right)$. Thus, carrying out the Fourier transform, we have

$$
\widehat{F_{A^{+}}^{* l m}}(\Xi)=\sum_{0 \leq j<k \leq 3} c_{j k} e^{-\imath\langle\varepsilon, a\rangle} \widehat{F_{A}^{j k}}\left({ }^{t} \Lambda^{-1} \Xi\right), \quad \Xi \in \mathbb{R}^{4},
$$

where $c_{j k^{\prime}}$ S are constants determined by $\Lambda$. Hence, $F_{A^{*}}^{*} \in \widetilde{S}\left(\Lambda^{-1} L\right)$ if $F_{A} \in \widetilde{S}(\mathbb{L})$. On the other hand, by virtue of $(5.1)$ we see that $\Lambda^{-1} G^{-1 t} \Lambda^{-1}=G^{-1}$. Then it follows that

$$
\left\langle{ }^{t} \Lambda^{-1} \Xi,{ }^{t} \Lambda^{-1} \Xi^{\prime}\right\rangle_{L M^{*}}=\left\langle\Xi, \Xi^{\prime}\right\rangle_{L M^{*}},
$$

where

$$
\left\langle\Xi, \Xi^{\prime}\right\rangle_{L M^{*}}:=\xi_{0} \xi_{0}^{\prime}-c^{2} \sum_{j=1}^{3} \xi_{j} \xi_{j}^{\prime}
$$

for $\Xi=\left(\xi_{0}, \cdots, \xi_{3}\right)$, etc. Thus, we can see that

$$
{ }^{t} \Lambda^{-1} D=D \text {. }
$$

Namely, the set $D$ in the dual space of the space-time is invariant under Poincare transformations in the space-time. We also see that $D_{1}$ in Proposition 1.4 is invariant.

After all we can conclude Theorems $1.2,1.3,1.5,1.7$ and Corollary 1.6 hold on any inertial frame.

In the last of this section we show that each field $F_{k}$ in Corollary 1.6 is regarded as a time-independent one on a suitable inertial frame. Let $V=V_{k}$ is timelike;

$$
V \in T:=\left\{(t, x) \in \mathbb{R}^{4} ; \quad c|t|>|x|\right\}
$$

with $\langle V, V\rangle_{L M}=c^{2}$. Then it is known that there exists a Lorentz transform $\Lambda$ : $\mathbb{R}_{t} \times \mathbb{R}_{x}^{3} \longrightarrow \mathbb{R}_{t^{\prime}} \times \mathbb{R}_{x^{\prime}}^{3}$ such that $\Lambda V={ }^{t}(1,0,0,0)$. Therefore $F_{A}\left(\Lambda^{-1} \cdot\right)$ is $t^{\prime}$. independent. 


\section{Acknowledgments}

The author would like to thank Prof. Luigi M. Ricciardi and the Department of Mathematics at Università di Napoli "Federico II " for their kind hospitality during his stay at Naples. He also would like to express his gratitude to Prof. Volker Enss for stimulating discussions.

\section{References}

[A1] Arians, S., Geometric approach to inverse scattering for the Schrodinger equation with magnetic and electric potentials, J. Math. Phys., 38(1997), 2761-2773.

[A2] - Geometric approach to inverse scattering for Hydrogen-like systems in a homogeneous magnetic field, preprint.

[E-We] Enss, V. and Weder. R., The geometrical approach to multidimensional inverse scattering, J. Math. Phys., 36 (1995), 3902-3921.

[F] Faddeev, L. D., Uniqueness of the inverse scattering problem, Vestn. Leningr. Univ.. 7 (1956), 126-130.

[H] Hormander, L., The Analysis of Partial Differential Operators I, Springer Verlag, 1989.

[Is] Isozaki, H., Inverse scattering theory for Dirac operators. Ann. Inst. H. Poincare, 66 (1997), 237-270.

[Is-K] Isozaki, H. and Kitada, H., Scattering matrices for two-body Schrödinger operators, Sci. Papers College Arts Sci. Unıv. Tokyo, 35 (1985), 81-107.

[It] Ito, H. T., High-energy behavior of the scattering amplitude for a Dirac operators, Publ. RIMS, Kyoto Univ., 31 (1995), 1107-1133.

[J] Jung, W.. Geometrical approach to inverse scattering for the Dirac equation, J. Math. Phys., 38(1997), 39-48.

[Ne] Newton, R. G., Inverse Schrodinger Scattering in Three Dimensional, Springer Verlag, 1989.

[Ni] Nicoleau, F., A stationary approach to inverse scattering for Schrödinger operators with first order perturbation, Comm. Part. Diff. Eq.. 22 (1997), 527-553.

[R.S] Ramm, A. G. and Sjostrand, J., An inverse problem for the wave equation, Math. Z., 206 (1991), 119-130.

[Sa] Saitō, Y., An asymptotic behavior of the S-matrix and the inverse scattering problem, $J$. Math. Phys., 25 (1984) , 3105-3111.

[St] Stefanov, P. D.. Uniqueness of the multi-dimensionals inverse scattering problem for time dependent potentials, Math. Z., 201 (1989), 541-559.

[Tha] Thaller, B.. The Dirac Equation, Springer Verlag, 1992.

$[\mathrm{Wa}$ ] Wang, X. P.. On the uniqueness of inverse scattering for $N$-body systems, Inverse Probl., 10 (1994), 765-784.

[We1] Weder, R., Inverse scattering for $N$-body systems with time-dependent potentials, in Inverse Problems of Wave Propagation and Diffraction, Eds. Chavent, G. and Sabatier, P. C., Lecture Notes in Phys. 486, Springer Verlag, 1997.

[We2] - Multidimensional inverse scattering in an electric field, J. Funcl. Anal., 139(1996), 441-465.

[We3] - Inverse scattering for the nonlinear Schrödinger equation, preprint 1997. 
